\begin{tabular}{|c|l|}
\hline Title & Detonation Transition A round Cylindrical Reflector of Pul se Detonation Engine Initiator \\
\hline Author(s) & Wakita, Masashi; Tamura, Masay oshi; Terasaka, A kihiro; Saj iki, Kazuya; Totani, Tsuy oshi; Nagata, Harunori \\
\hline Citation & $\begin{array}{l}\text { Journal of Propulsion and Power, 29(4), 825-831 } \\
\text { https:/doi.org/L.2514/L.B34704 }\end{array}$ \\
\hline Issue Date & 2013 07 \\
\hline Doc URL & http://hdl.handle.net/2115/53362 \\
\hline Type & article (author version) \\
\hline File Information & 20130107 wakita.pdf \\
\hline
\end{tabular}

Instructions for use 


\title{
Detonation transition around cylindrical reflector of pulse detonation engine initiator
}

\author{
Masashi Wakita ${ }^{1}$, Masayoshi Tamura ${ }^{2}$, Akihiro Terasaka ${ }^{3}$, Kazuya Sajiki $^{4}$, \\ Tsuyoshi Totani ${ }^{5}$ and Harunori Nagata ${ }^{6}$ \\ Hokkaido University, Hokkaido 060-8628, Japan
}

To achieve reliable transmission of detonation waves to a pulse detonation engine (PDE) combustor (detonation chamber), the authors propose a PDE initiator that uses a cylindrical reflector downstream of a predetonator exit. The detonation wave propagates around the reflector to change the wave shape in three transition stages: from a planar detonation wave in the predetonator to an expanding cylindrical detonation wave, from the cylindrical wave to a planar toroidal detonation wave, and from the toroidal wave to a planar detonation wave in the detonation chamber. The cylindrical wave propagates along a cylindrical path between the reflector and front wall of the detonation chamber, and the toroidal wave propagates along an annular path between the reflector and sidewall of the detonation chamber. The purpose of this study was to examine the effect of the gap width $L$ of the annular path on the transition stages from cylindrical to toroidal and from toroidal to planar. A series of experiments that filled the entire test section with the driver gas mixture (stoichiometric hydrogen-oxygen mixture) showed that the expanding cylindrical detonation wave was sufficiently strong to survive the rarefaction waves from the corners of the reflector at all of the investigated annular gap widths $(5,10,15$, and $20 \mathrm{~mm})$ and was transmitted to the planar toroidal wave successfully in all cases. When the strength of the cylindrical detonation wave was under a super-critical condition for diffraction at the reflector corner, the necessary filling distance for the driver gas was predicted well by the Whitham theory. A second series of experiments showed the effect of the annular gap width on the detonation transition from the planar toroidal detonation wave to the planar detonation wave. Two different

\footnotetext{
${ }^{1}$ Assistant Professor, Graduate School of Engineering, Division of Mechanical and Space Engineering, North 13, West 8, Kita-ku, Sapporo 060-8628, Japan; m-wakita@eng.hokudai.ac.jp, Member AIAA.

${ }^{2}$ Graduate Student, Graduate School of Engineering, Division of Mechanical and Space Engineering, North 13, West 8, Kita-ku, Sapporo 060-8628, Japan.

${ }^{3}$ Graduate Student, Graduate School of Engineering, Division of Mechanical and Space Engineering, North 13 , West 8, Kita-ku, Sapporo 060-8628, Japan.

${ }^{4}$ Graduate Student, Graduate School of Engineering, Division of Mechanical and Space Engineering, North 13, West 8, Kita-ku, Sapporo 060-8628, Japan.

${ }^{5}$ Associate Professor, Graduate School of Engineering, Division of Mechanical and Space Engineering, North 13, West 8, Kita-ku, Sapporo 060-8628, Japan; tota@eng.hokudai.ac.jp, Member AIAA.

${ }^{6}$ Professor, Graduate School of Engineering, Division of Mechanical and Space Engineering, North 13, West 8, Kita-ku, Sapporo 060-8628, Japan; nagata@eng.hokudai.ac.jp, Member AIAA.
} 


\section{types of detonation transitions termed "continuous transition" and "temporal quenching" were observed. The threshold value of $L / \lambda$ for "continuous transition" is approximately 4.}

\section{Introduction}

A pulse detonation engine (PDE) in which propellant burns in intermittent detonation waves has attracted attention because of its simplicity and theoretically high thermal efficiency [1-4]. A major issue that needs to be resolved for practical use of the PDE is "detonation initiation." When a PDE operates in air-breathing mode, the combustible gas is likely to be a fuel-air mixture, and its detonation sensitivity of the combustible mixture is lower than that of fuel-oxygen mixtures [5]. Another case where sensitivity may be low is when the fuel is in the liquid phase. The energy required to initiate detonation of low-sensitivity propellants such as the fuel-air mixture and liquid phase mixture mentioned above is too much to directly initiate a detonation wave using commonly employed energy sources [4].

To initiate a detonation wave using commonly employed energy sources, a deflagration-to-detonation-transition (DDT) process is commonly employed. However, a low-sensitivity combustible mixture needs both a long distance and time for the DDT process, which results in decreases in operating frequency and thermal efficiency. A number of methods for reducing the DDT length and time have been reported that use vortex generators such as the Shchelkin spiral in the upstream region of the detonation chamber. However, vortex generators in a detonation chamber may result in irregular ignition due to temperature increases during operation.

Another detonation initiation method is the "predetonator concept." Here, a detonation wave is readily initiated in a small-diameter tube (predetonator) using a low-energy source, and the detonation wave is transmitted into a larger-diameter detonation chamber containing a low-sensitivity mixture of propellants (the target gas) [4]. Knowledge of the particulars of the detonation transition brought about by abrupt area changes from the predetonator to the main chamber is of importance in detonation studies, and many investigations have addressed this issue [6-9]. It has been shown that the tube diameter $\boldsymbol{d}$ must be at least 13 times the cell size $\lambda$ for a successful detonation transition [10-12]. However, subsequent experimental studies showed that $\boldsymbol{d}_{\boldsymbol{c}}=13 \boldsymbol{\lambda}$ is not realized, as reviewed in [13]. Many methods for enhancing the detonation transition at the abrupt change of area have been proposed, and typical methods include the use of 1) shock reflection and shock-focusing devices [14-16] and 2) a cone-shaped exit with a gradual area change that reduces the lateral expansion [12, 17-19]. 
Most detonation initiators use a driver gas mixture in the uppermost part of the tube: typically, hydrogenoxygen or ethylene-oxygen. The short DDT length (or time) of these mixtures makes it possible to use an extremely short predetonator, and using a driver gas mixture improves the ignition probability and reliability of a PDE system. However, the additional oxygen in the driver gas causes an increase in the onboard propellant weight and reduces the specific impulse $\left(\mathrm{I}_{\mathrm{sp}}\right)$ of the PDE system. A performance analysis by Bussing et al. showed that a driver gas accounting for only $1 \%$ of the total combustion chamber volume results in a $22 \%$ reduction in $I_{\mathrm{sp}}[20,21]$. Accordingly, it is desirable to minimize the driver gas usage since the amount of the driver gas is proportional to the sectional area of the predetonator and $\mathrm{I}_{\mathrm{sp}}$ increases as the diameter of the predetonator decreases.

To realize a successful transition of detonation at the predetonator exit in a PDE operating in air-breathing mode, in which the combustible gas is a fuel-air mixture, the authors propose a PDE initiator that uses a reflecting board near the exit of the predetonator tube, as shown in Fig. 1 [22, 23]. With the reflecting board, the detonation wave propagates around the reflecting board, and the wave shape change over three transition stages, as suggested in Fig. 2: from planar (A) to cylindrical (B), toroidal (C), and back to planar again (D). Previous research [22] showed that the transition from the incident planar detonation wave (Fig. 2-A) to an expanding cylindrical detonation wave (Fig. 2-B) in the first transition process is a sufficient condition for successful detonation wave propagation in the detonation chamber. Successful transition to the expanding cylindrical detonation wave (Fig. 2-B) occurs when the predetonator diameter $\boldsymbol{d}$ is larger than $6.3 \lambda$ and the reflecting board distance $\boldsymbol{w}$ equals the predetonator diameter $\boldsymbol{d}$. Inspired by a previous result where the transition to an expanding cylindrical detonation wave was found to be a sufficient condition for a planar detonation wave (Fig. 2-D) to propagate in the detonation chamber, the authors further examined the situation experimentally with a "reflecting board" combined with "overfilling" of driver gas, as shown in Fig. 3 [23]. By using the configuration in Fig. 3, the authors achieved a successful transition to the planar detonation wave (Fig. 2-D) when the overfilling distance $\boldsymbol{h}_{\text {overfil }}$ was at least $33.2 \mathrm{~mm}$. When $\boldsymbol{h}_{\text {overfill }}$ was $33.2 \mathrm{~mm}$, the expanding cylindrical detonation wave (Fig. 2-B) transformed not to an imploding toroidal detonation wave (Fig. 2-C) but to a torus-shaped pressure wave because of the mixing effect of the driver gas and target gas around the reflecting board. The torus-shaped pressure wave concentrated at the axis of the detonation chamber behind the reflecting board (right side in Fig. 3) to generate a region of high temperature and high pressure sufficient for detonation re-initiation. Additionally, a 100-mm-long cylindrical reflector with an $80 \mathrm{~mm}$ diameter, as shown in Fig. 
4-1, was found to prevent the mixing effect of the driver gas and the target gas and make it possible to reduce the overfilling distance $\boldsymbol{h}_{\text {overfil }}$ to $17.2 \mathrm{~mm}$.

In the cylindrical reflector configuration (Fig. 4), usage of the driver gas and the success or failure of the transition from the cylindrical detonation wave to the toroidal detonation wave are strongly influenced by the annular gap width $\boldsymbol{L}$ (gap between the chamber wall and the reflector, as shown in Fig. 3). In the previous study, the annular gap width $\boldsymbol{L}$ was fixed at $10 \mathrm{~mm}$; the present study examined the effect of the annular gap width $\boldsymbol{L}$ on the detonation transition processes from the expanding cylindrical detonation wave to the toroidal detonation wave in the driver gas mixture and clarified the relation between the annular gap width $\boldsymbol{L}$ and required overfilling distance. This study also examined the effect of changes in the annular gap width $\boldsymbol{L}$ on the transition processes from the toroidal detonation wave to the planar detonation wave and classified the process according to the $L / \lambda$ parameter (ratio between the annular gap and cell size).

\section{Experimental details}

Two experimental configurations were used in this study. The left schematic in Fig. 4 shows the short chamber configuration, which was employed to show the effect of the annular gap width $\boldsymbol{L}$ on the detonation transition from the cylindrical detonation wave to the toroidal detonation wave. It consisted of a predetonator, detonation chamber, and cylindrical reflector (at II in the detonation chamber). The detonation chamber was $280 \mathrm{~mm}$ long and had an internal diameter of $100 \mathrm{~mm}$. The predetonator upstream of the detonation chamber was $540 \mathrm{~mm}$ long and has an internal diameter of $10 \mathrm{~mm}$. This length was sufficiently long compared with the DDT length of a stoichiometric hydrogen-oxygen mixture for this tube diameter. The annular gap width $\boldsymbol{L}$ was varied from $5 \mathrm{~mm}$ to $20 \mathrm{~mm}$ in increments of $5 \mathrm{~mm}$ by changing the diameter of the $100 \mathrm{~mm}$ long cylindrical reflector. In this experiment, the test gas mixture was a stoichiometric hydrogen-oxygen mixture.

The right schematic in Fig. 4 shows the long chamber configuration used to investigate the effect of $\boldsymbol{L}$ on the detonation transition from a cylindrical wave to a planar detonation wave. The predetonator was the same as in the short configuration, and the lengths of the detonation chamber and cylindrical reflector were 620 and $230 \mathrm{~mm}$, respectively. Pressure histories were obtained at M1-M4, and an overfilling method was employed to demonstrate the propagation of the detonation from the driver gas to the target gas during the annular path. To overfill the driver 
gas in the combustion chamber, an additional volume was connected with the predetonator via a ball valve, as shown in Fig. 4. Figure 5 depicts the overfilling procedure. Initially, the valve in the predetonator is closed and the driver gas mixture and target gas mixture fill the areas upstream and downstream of the valve, respectively (Fig. 5(A)). The gases are completely mixed prior to the two chambers being filled. The pressure of the driver gas $\boldsymbol{p}_{1}$ is higher than that of the target gas $\boldsymbol{p}_{2}$; and when the valve opens (Fig. 5-(B)), the driver gas overfills to position $\boldsymbol{h}$, and a balanced pressure $\boldsymbol{p}_{3}$ is established. In the experiments, $\boldsymbol{h}$ was chosen to be $30 \mathrm{~mm}$. The driver gas mixture was a stoichiometric hydrogen-oxygen mixture, and the target gas mixtures were stoichiometric hydrogen-oxygen mixtures diluted with nitrogen.

Common to all experiments, the initial pressure, the balanced pressure $\boldsymbol{p}_{3}$, was $1 \mathrm{~atm}$. The cylindrical reflector was supported by four shafts connected to the upper flange of the detonation chamber. The reflector clearance, which is the distance from the predetonator exit to the upstream surface of the cylindrical reflector, was set to 10 $\mathrm{mm}$. This is the necessary minimum value for a successful transition from the planar detonation wave in the predetonator to the cylindrical detonation propagating upstream of the reflector in a stoichiometric hydrogenoxygen atmosphere $[22,23]$. A control device determined the timing of the valve opening and ignition and activated a spark plug $1 \mathrm{~s}$ after the valve opened. Soot foils collected tracks of the triple points of detonation waves at four locations, which are shown in Fig. 4: the sidewall of the detonation chamber (I and III) and at the cylindrical reflector (II and IV).

\section{Results and Discussion}

\section{Effect of annular gap width on the detonation transition from cylindrical to toroidal wave}

A series of experiments with the driver gas mixture (stoichiometric hydrogen-oxygen mixture) completely filling the combustion chamber showed the effect of the annular gap width $\boldsymbol{L}$ on the cylindrical-to-toroidal detonation transition. Figure 6 shows photos of soot tracks on sections of the sidewalls of the detonation chamber and the cylindrical reflector with annular gap widths $\boldsymbol{L}$ of 5, 10, 15, and 20 mm. The combustion wave traveled from left to right in the figures. The expanding cylindrical detonation wave passing through the coaxial gap upstream of the reflector collided with the far left of the detonation chamber soot tracks and turned

to the right. The boundary lines (first) and (second) in the $\boldsymbol{L}=5 \mathrm{~mm}$ tracks were commonly observed in all 
cases. The boundary line (third) was observed only on the sidewall of the detonation chamber of $\boldsymbol{L}=5 \mathrm{~mm}$. These boundary lines indicated the positions of detonation wave reflection. The left side of the boundary line (I) is a black zonal area. At this location, the expanding cylindrical detonation wave impacted the sidewall normally. The width of the area was approximately $10 \mathrm{~mm}$ in all cases, which is equal to the reflector clearance. Figure 7 is a schematic representation of the progress of the expanding cylindrical detonation wave diffracting from the edge of the reflector board to the downstream. Observations of the chamber and reflector walls, as shown in Fig. 6, indicated that the expanding cylindrical wave did not decay by the rarefaction waves from the corner of the reflector (line OC in Fig. 7) and maintained its strength while passing across the annular gap width $\boldsymbol{L}$. Because the cell size $\lambda$ of a stoichiometric hydrogen-oxygen mixture is about 1.2-1.3 $\mathrm{mm}$ at ambient pressures, the reflector clearance here corresponded to $8-9 \lambda$. An earlier study established that when a detonation wave transits from a rectangular channel to an unconfined space, the critical width of the flow channel is $10 \lambda[10]$. Compared with half the value of $10 \lambda$, the results here suggest that a value of $8-9 \lambda$ is large enough for the expanding cylindrical detonation wave to survive the rarefaction waves. Therefore, this condition would be a supercritical condition for the transition of the cylindrical detonation wave.

On the right side of the boundary line (first) in Fig. 6, fine cellular structures were observed in all cases. These structures indicate that the expanding toroidal detonation wave was reinitiated at the line (first) due to the strong reflection of the cylindrical detonation wave on the sidewall. On the left side of the boundary at line (second), no cellular structures were observed. This line (second) corresponds to the interface between the diffracted shock wave spreading along the sidewall of the reflector and the expanding toroidal detonation wave from the sidewall of the detonation chamber. A further reflection (third boundary line) was observed on the detonation chamber wall for the $\boldsymbol{L}=5 \mathrm{~mm}$ case only. The distance from the upstream edge of the cylindrical body to the boundary line (second) strongly depended on the annular gap width $\boldsymbol{L}$, and the distance was almost equal to the gap width. These experimental results suggest that angle $\boldsymbol{\theta}$ in Fig. 7 is approximately $45^{\circ}$ regardless of the gap width $\boldsymbol{L}$.

The Whitham theory [24] was applied to qualitatively evaluate the diffraction of the cylindrical detonation wave. The wall Mach number $M_{w}$ with the angle of the diffracted corner $\beta$ is given by 
$M_{w}=a M_{c j}$

where $a=\exp (\beta / \sqrt{n})$ and $n=\frac{\gamma+2}{\gamma}+\sqrt{\frac{2 \gamma}{\gamma+1}}$.

$M_{c j}$ is the Mach number of the cylindrical wave and $\gamma$ is the ratio of the specific heats. From the initial condition of the stoichiometric $\mathrm{H}_{2}-\mathrm{O}_{2}$ mixture, $\gamma=1.13, M_{\mathrm{cj}}=5.2$, and $\beta=-\pi / 2$. Thus, $a=0.447$ and $M_{\mathrm{w}}=2.3$. The soot tracks show that the width of the cylindrical detonation wave $(w=10 \mathrm{~mm})$ did not change from the exit to the detonation chamber sidewall. Therefore, $\triangle \mathrm{OAB}$ in Fig.7 became a right-angled triangle. Assuming that the Mach number of the detonation wave along $\mathrm{OA}$ and $\mathrm{AB}$ was $M_{c j}$ and that along $\mathrm{OB}$ was $M_{w}$, the angle $\theta$ of $\mathrm{OAB}$ is given by

$\cos \theta=\frac{1-a^{2}}{1+a^{2}}$ and $\theta=48.1^{\circ}$

This result shows that the Whitham theory qualitatively agreed with the experimental results. Angle $\boldsymbol{\theta}$ depended only on the ratio $\boldsymbol{a}$, which depends only on the physical properties of the driver gas if the corner angle is given. If the formation of a planar toroidal detonation wave propagating in the driver gas section is a sufficient condition for successful transformation to a planar toroidal detonation wave propagating in the target gas composition, it is only necessary to supply the driver gas mixture to the vicinity of the boundary line (second). At this point, the expanding toroidal detonation wave has completed much of the change to become a planar toroidal detonation wave. Therefore, the Whitham theory provides a good indication for the overfilling distance $\left(\boldsymbol{w}+\boldsymbol{l}_{2}\right)=\boldsymbol{w}+\boldsymbol{L} \tan \boldsymbol{\theta}$ when the strength of the cylindrical detonation wave is under a supercritical condition for diffraction of the reflector corner.

Experiments with hydrogen-oxygen driver gas mixtures overfilling into the hydrogen-air target gas mixtures were conducted by using the short chamber configuration. These experiments showed that the critical overfilling distance was around $30 \mathrm{~mm}$ regardless of the annular gap width $\boldsymbol{L}$. The actual necessary overfilling distances were longer than the values expected by the Whitham theory because of the mixing effect of the driver gas mixture and target gas mixture at the contact surface. Figure 8 shows soot tracks on the sidewall of 
the detonation chamber and cylindrical reflector under the critical condition. Common to all gap widths, the positions of the first reflection $l_{1}$ and second reflection $l_{2}$ corresponded to those shown in Fig. 6 . The expanding toroidal waves commenced at the first reflection positions and transformed to the planar toroidal waves at the second reflection position. These planar toroidal waves were transmitted without quenching in the target gas area downstream of $30 \mathrm{~mm}$. When $\boldsymbol{L}=5,10$, and $15 \mathrm{~mm}$, the cell sizes of the wave propagating in the target gas area were much smaller than that of the stoichiometric hydrogen-air mixture, which was about $10 \mathrm{~mm}$ at ambient pressures. These results suggest that the detonation wave at the exit of the annular path was an overdriven detonation. Unfortunately, downstream of the back face of the reflector, the planar toroidal wave was quenched by the rarefaction wave from the downstream edge of the reflector. The quenching line shifted downstream with increasing gap width. Finally, a successful transition to a planar detonation wave downstream of the chamber was only observed when $\boldsymbol{L}=10$ and $15 \mathrm{~mm}$. These planar detonation waves were possibly reinitiated by the shock wave implosion downstream of the reflector.

\section{Effect of the annular gap width on the detonation transition from toroidal to planar waves}

As detailed above, the planar detonation wave at the exit of the annular path became an overdriven detonation in some cases. To obtain the steady planar toroidal detonation wave, the long chamber configuration—which had a 230-mm-long annular path as shown in Fig. 4-was used. In these experiments, the overfilling distance of the stoichiometric hydrogen-oxygen driver mixture was set to $30 \mathrm{~mm}$ in all cases. Figure 9 shows the cell size $\lambda$ plotted with data obtained in other investigations. The cell size $\lambda$ of the present study was measured 10 $\mathrm{mm}$ upstream from the aft end of the annular path, and the horizontal axis represents the nitrogen concentration of the target gas mixture. For the stoichiometric hydrogen-air mixture, the nitrogen concentration was 55.6\%, and the cell size is about $10 \mathrm{~mm}$. The present data are in good agreement with the previous data throughout the concentration range. Figure 10 represents the ratio of the detonation velocity $\boldsymbol{D}$ and theoretical value $\boldsymbol{D}_{c j}$ with respect to the nitrogen concentration. The detonation velocity $\boldsymbol{D}$ was measured between M1 and M2 in Fig. 1 and the theoretical detonation velocity at the Chapman-Jouguet (CJ) state was calculated by the NASA computer program Chemical Equilibrium with Applications (CEA). The present data also agreed well with the theoretical values at nitrogen concentration of $0 \%-60 \%$ of nitrogen concentration. Above a nitrogen 
concentration of $60 \%$, the cell size of the detonation wave exceeded the annular gap width and a steady planar toroidal detonation wave was not obtained.

Figure 11 shows photos of the soot tracks on the sidewalls of the detonation chamber and pressure histories. The nitrogen concentrations of the target gas mixture were $20 \%, 30 \%$, and $32.5 \%$ from the top. The vertical broken line at $240 \mathrm{~mm}$ represents the downstream edge of the annular section. At $20 \%$ and $30 \%$, there was a regular cell pattern with the cell size increasing at $30 \% \mathrm{~N}_{2}$ downstream of the exit due to the rarefaction wave from the corner; however, the detonation wave was maintained. Such results were classified as "continuous transition." When the target gas mixture had $32.5 \% \mathrm{~N}_{2}$, the detonation wave was temporarily quenched at the exit by the rarefaction wave; this was classified as "temporal quenching." Figure 12 shows the kinds of transitions for various annular gap widths: circles and triangles represent continuous transition and temporal quenching, respectively, and the vertical axis represents the nitrogen concentration. When the annular gap width was equal to $5 \mathrm{~mm}$, temporal quenching was observed even at a nitrogen concentration of $0 \%$; no continuous transition cases were observed in the range of experimental conditions examined in the present study. The continuous transition limits depended on the gap width and the threshold value became larger as the gap width was increased. The kinds of transitions for the various annular gap widths shown in Fig. 12 were reevaluated by the $L / \lambda$ ratio, and the results are shown in Fig. 13 . The $L / \lambda$ threshold value for continuous transition was approximately 4. Accordingly, the critical annular gap width for successful transition of detonation wave may be determined as $4 \lambda$. The exit of the annular path in the experiments was similar to a rectangular outlet in that one side was a wall and the other was unconfined space. An earlier study showed that when a detonation wave transits from a rectangular outlet to unconfined space, the critical width of the flow channel is $10 \lambda[10]$; later studies showed that the critical width is a function of the aspect ratio of the exit cross section and that the critical width decreases from $10 \lambda$ to $3 \lambda$ as the aspect ratio increases towards infinity [25, 26]. In this study, the toroidal detonation wave at the exit expanded only toward the inside of the chamber and not toward the outside. Compared with the half value of the critical width of $10 \lambda$ to $3 \lambda$ for the rectangular outlet, the critical gap width of $4 \lambda$ determined for the annular exit here appears to agree well with the previous results. One of the differences between the rectangular outlet and annular exit here is the curvature of the cross 
section. In this study, only the inner curvature of the annular exit was changed by changes in the diameter of the reflector. However the effect of the inner curvature on the detonation transition status was not investigated.

\section{Conclusion}

Two series of experiments were performed on the detonation transition from an expanding cylindrical detonation wave to a planar toroidal detonation wave around a cylindrical reflector to show the effects of the annular gap width of the annular flow passage.

When a driver gas mixture (stoichiometric hydrogen-oxygen mixture) filled the entire test section the expanding cylindrical detonation wave was sufficiently strong to survive the rarefaction waves from the corner of the reflector at all annular gap widths $(5,10,15$, and $20 \mathrm{~mm})$, and the distance from the upstream reflector

edge necessary for the cylindrical detonation wave to completely change to a planar cylindrical detonation wave was equivalent to the annular gap width. When the strength of the cylindrical detonation wave was under a supercritical condition for diffraction at the reflector corner, this distance was predicted well by the Whitham theory.

In tests on the effect of the annular gap width on the detonation transition from a planar toroidal detonation wave to a planar detonation wave in the long chamber configuration, two different types of detonation transitions were observed: continuous transition and temporal quenching. The threshold value of $L / \lambda$ (annular gap width vs. cell size ratio) for continuous transition was approximately 4; thus the critical annular gap width for successful transition of the detonation wave was $4 \lambda$. The exit of the annular path in the present experimental set-up was similar to a rectangular outlet, with one side a wall and the other unconfined space. Compared with the half value of the critical widths of $10 \lambda$ and $3 \lambda$ for rectangular outlets with aspect ratios of 1 and infinity, the critical gap width of $4 \lambda$ for the present annular exit determined agreed well with other reports.

\section{Acknowledgments}

This research was supported by a JSPS Grants-in-Aid for Young Scientists (B) (KAKENHI) (21760646) 


\section{References}

[1] Nicholls, J. A., Wilkinson,H. R., and Morrison, R. B., "Intermittent Detonation as a Thrust-Producing Mechanism," Jet Propulsion, Vol. 27, No. 5, 1957, pp. 534-541.

[2] Kailasanath, K., "Review of Propulsion Applications of Detonation Waves," AIAA Journal, Vol. 38, No. 9, 2000, pp. 1698-1708.

[3] Kailasanath, K., "Recent Developments in the Research on Pulse Detonation Engines," AIAA Journal, Vol. 41, No. 2, 2003, pp. 145-159.

[4] Roy, G. D., Frolov, S. M., Borisov, A. A., and Netzer, D. W., "Pulse detonation propulsion: challenges, current status, and future perspective," Progress in Energy and Combustion Science, Vol. 30, No. 6, 2004, pp. 545-672.

[5] Kaneshige, M., and Shepherd, J. E., "Detonation Database," Graduate Aerospace Laboratories of the California Inst. of Technology TR FM97-8, Pasadena, CA, 1997.

[6] Helman, D., Shreeve, R.P., and Eidelman, S., "Detonation Pulse Engine," AIAA Paper 86-1683, Jun. 1986.

[7] Edwards, D. H., Thomas, G. O. and Nettleton, M. A., "The diffraction of a planar detonation wave at an abrupt area change," Journal of Fluid Mechanics., Vol. 95, No. 1, 1979, pp. 79-96.

[8] Shepherd, J. E., Schultz, E., and Akbar, R., "Detonation Diffraction," Proceedings of the TwentySecond International Symposium on Shock Waves, Vol. 1, London, 2000, pp. 41-48.

[9] Pitgen, F., and Shepherd, J. E., "Detonation Diffraction in Gases," Combustion and Flame, Vol. 156, No. 3, March 2009, pp. 665-677.

[10] Mitrofanov, V. V. and Soloukhin, R. I., "The diffraction of multifront detonation waves," Soviet Physics-Doklady, Vol. 9, No. 12, 1965, pp. 1055-1058.

[11] Matsui, H., and Lee, J. H., "On the Measure of the Relative Detonation Hazards of Gaseous FuelOxygen and Air Mixtures," Proceedings of 17th Symposium (International) on Combustion, Combustion Institute, Pittsburgh, 1978, pp. 1269-1280. 
[12] Knystautas, R., Lee, J. H., and Guirao, C. M., "The Critical Tube Diameter for Detonation Failure in Hydrocarbon-Air Mixtures," Combustion and Flame, Vol. 48, No. 1, 1982, pp. 63-83.

[13] Schultz, E., "Detonation Diffraction Through an Abrupt Area Expansion," Ph.D. Thesis, California Inst. of Technology, Pasadena, CA, April 2000.

[14] Moen, O., Funk, J. W., Ward, S. A., Rude, M. G., and Thibault, P. A., "Detonation Length Scales for Fuel-Air Explosives," Progress in Astronautics and Aeronautics, Vol. 94, AIAA, New York, 1983, pp. $55-79$.

[15] Jackson, S. I., and Shepherd, J. E., "Detonation initiation in a tube via imploding toroidal shock waves," AIAA Journal, Vol.46, No. 9, 2008, pp. 2357-2367.

[16] Sorin R., Zitoun R., Khasainov B., and Desbordes D., "Detonation diffraction through different geometries," Shock Waves, Vol.19, No. 1, 2009, pp.11-23.

[17] Thomas, G. O., Edwards, D. H., Lee, J. H., Knystautas, R., Moen, I. O., Wei, Y. M., "Detonation diffraction by divergent channels. Dynamics of Explosions," Progress in Astronautics and Aeronautics, 1986, 106, pp144-154.

[18] Thomas, G. O., Williams, R. L., Detonation interaction with wedges and bends, Shock Waves, 2002, Vol. 11, No. 6, pp. 481-492.

[19] Khasainov, B., Presles, H. N., Desbordes, D., Demontis, P., and Vidal, P., "Detonation diffraction from circular tubes to cones," Shock Waves, Vol. 14, No. 3, 2005, pp. 187-192.

[20] Aamio, M.J., Hinkey, J.B., and Bussing, T.R.A., "Multiple Cycle Detonation Experiments During the Development of a Pulse Detonation Engine," AIAA Paper 96-3263, 1996.

[21] Brophy, C. M., Netzer, D. W., Sinibaldi, J., and Johnson, R., "Detonation of A JP-10 Aerosol for Pulse Detonation Application," High-Speed Deflagration and Detonation, ElEX-KM Publishers, Moscow, 2001.

[22] Wakita, M. Numakura, R. Itoh, Y. Sugata, S. Totani, T. Nagata, H., "Detonation Transition Limit at an Abrupt Area Change Using a Reflecting Board," Journal of Propulsion and Power, Vol. 23, No. 2, 2007, pp. 338-344. 
[23] Wakita, M. Numakura, R. Asada, T. Tamura, M. Totani, T. Nagata, H., "Driver Gas Reduction Effect of Pulse-Detonation-Engine Initiator Using Reflecting Board,” Journal of Propulsion and Power, Vol. 27, No. 1, 2011, pp. 162-170.

[24] Whitham, G. B., "A new approach to problems of shock dynamics Part I Two-dimensional problems," Journal of Fluid Mechanics, Vol. 2, 1957, pp. 145-171.

[25] Liu, Y. K., Lee, J. H. and Knystautas, R., "Effect of geometry on the transmission of detonation through an orifice," Combustion and Flame, Vol. 56, No. 2, 1984, pp. 215-225.

[26] Benedick, W. B., Knystautas, R., and Lee., J.H.S., "Large-scale experiments on the transmission of fuelair detonations from two-dimensional channels," Progress in Astronautics and Aeronautics, Vol. 94, 1984, pp. 546-555. 


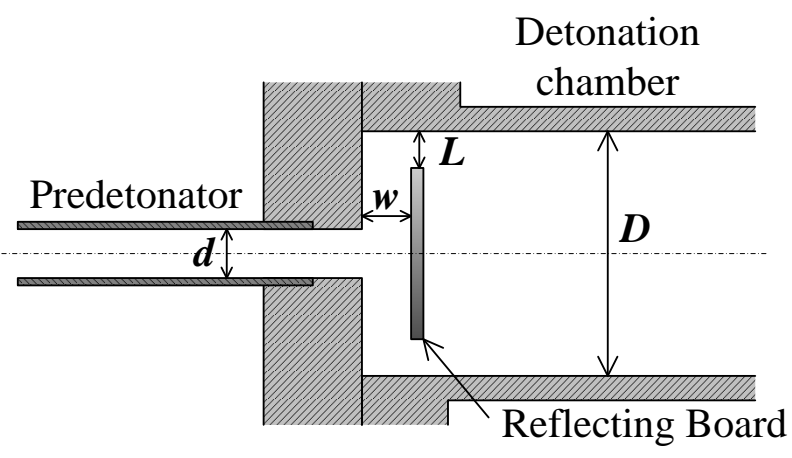

Fig. 1 Test section

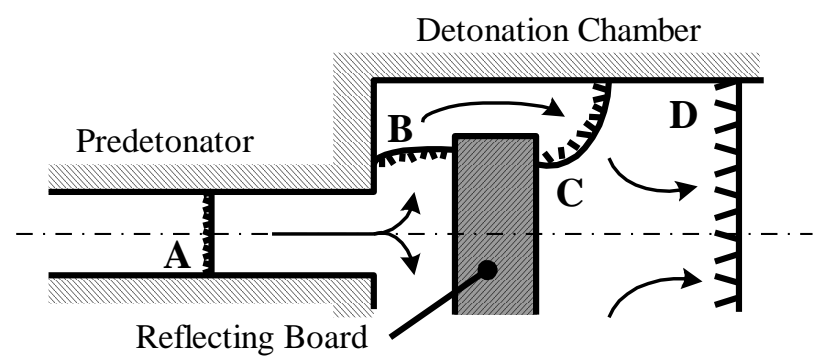
A. Planar detonation wave
B. Expanding cylindrical detonation wave
C. Imploding toroidal detonation wave
D. Planar detonation wave

Fig. 2 Transition of detonation waves

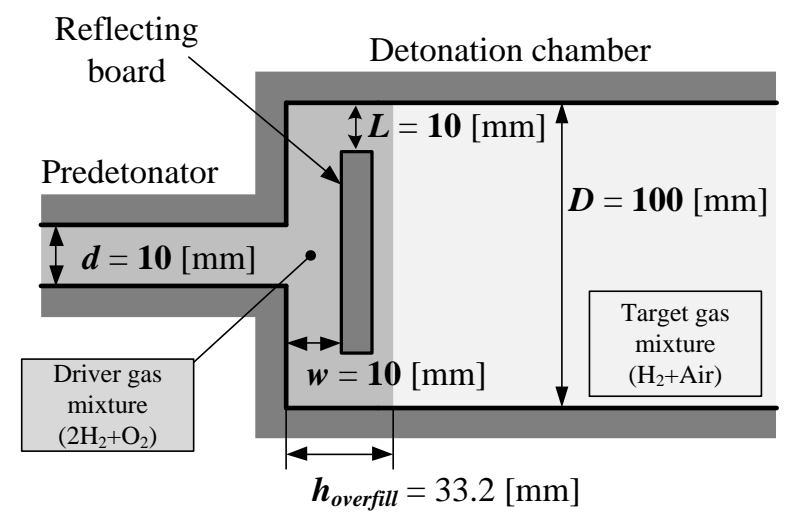

Fig. 3 Transition of detonation waves 


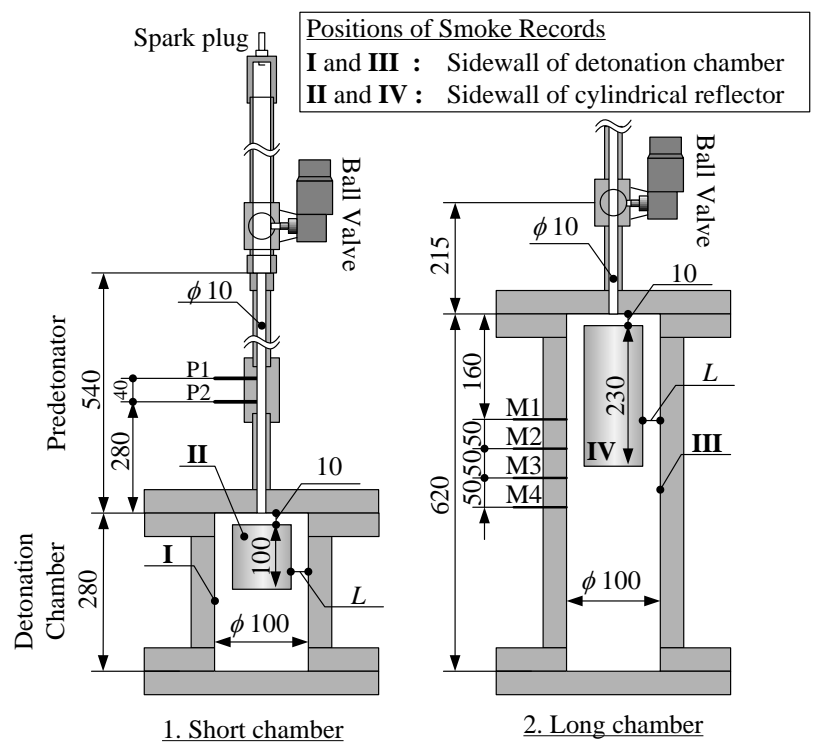

Fig. 4 Experimental setup

(A) Valve close (B) Valve open

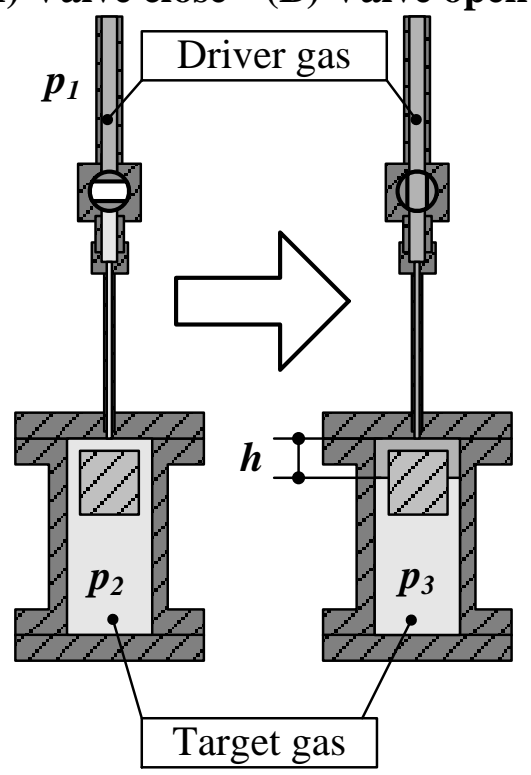

Fig. 5 Overfilling arrangement 

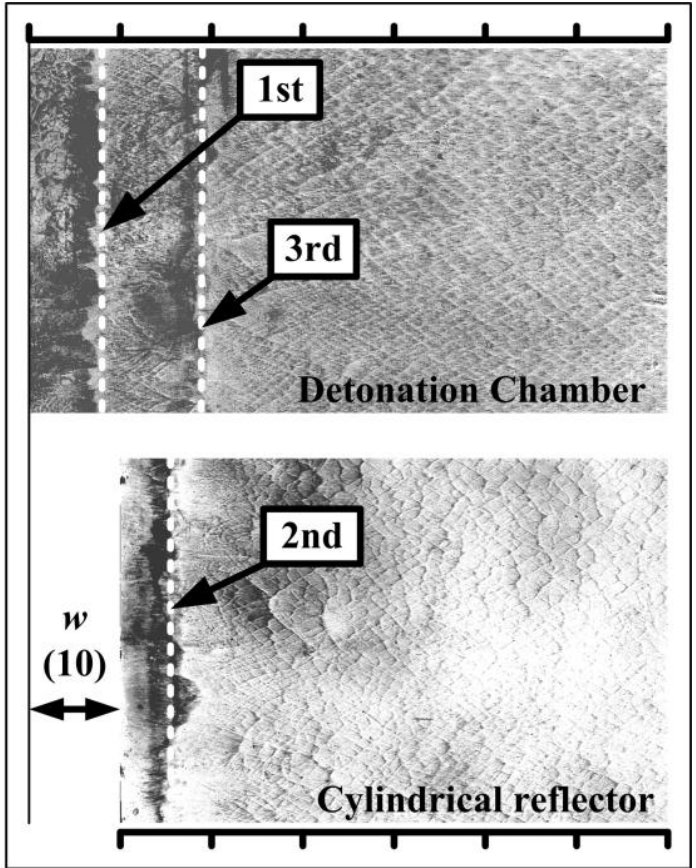

$$
\boldsymbol{L}=5[\mathrm{~mm}]
$$

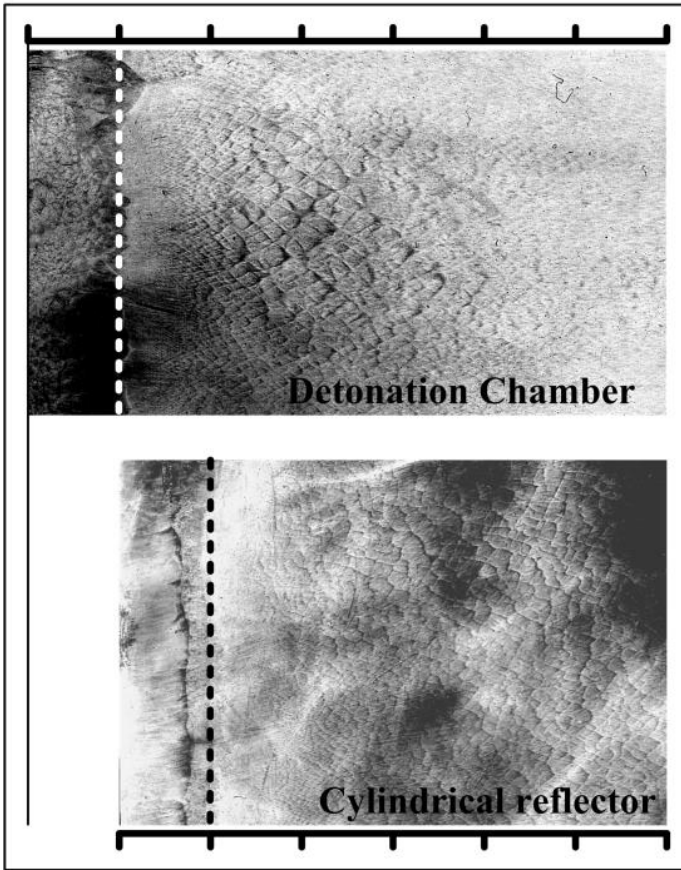

$\boldsymbol{L}=10[\mathrm{~mm}]$ 

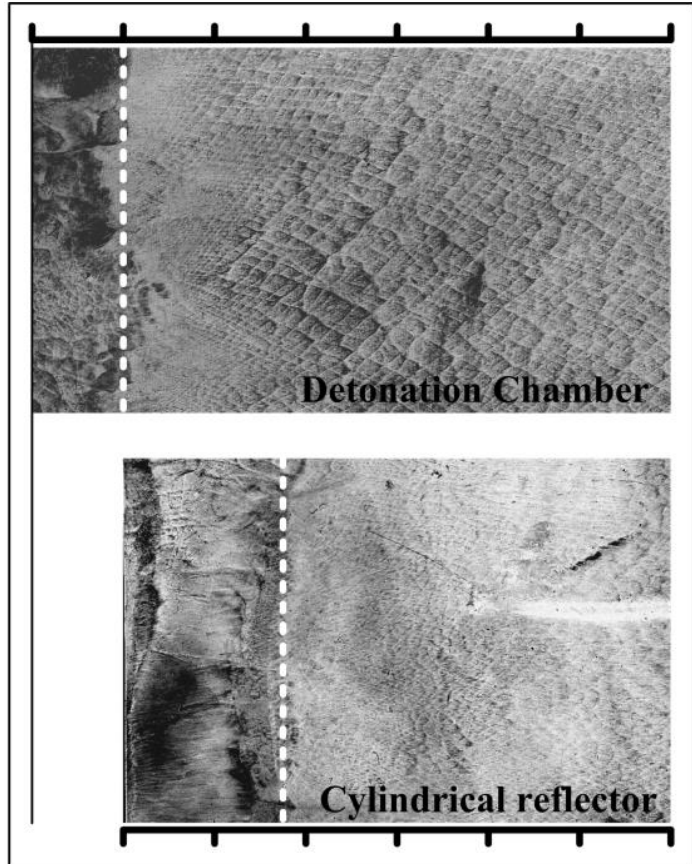

$\boldsymbol{L}=15[\mathrm{~mm}]$
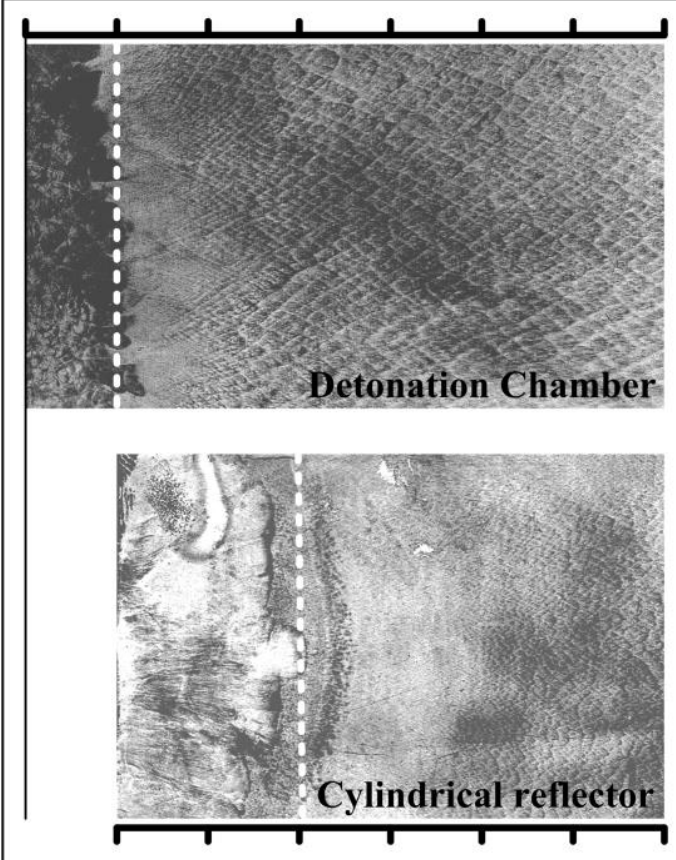

$\boldsymbol{L}=20[\mathrm{~mm}]$

Fig. 6 Soot tracks on the detonation chamber and cylindrical reflector walls with annular gap widths $\boldsymbol{L}$ of $5,10,15$, and $20 \mathrm{~mm}$. 


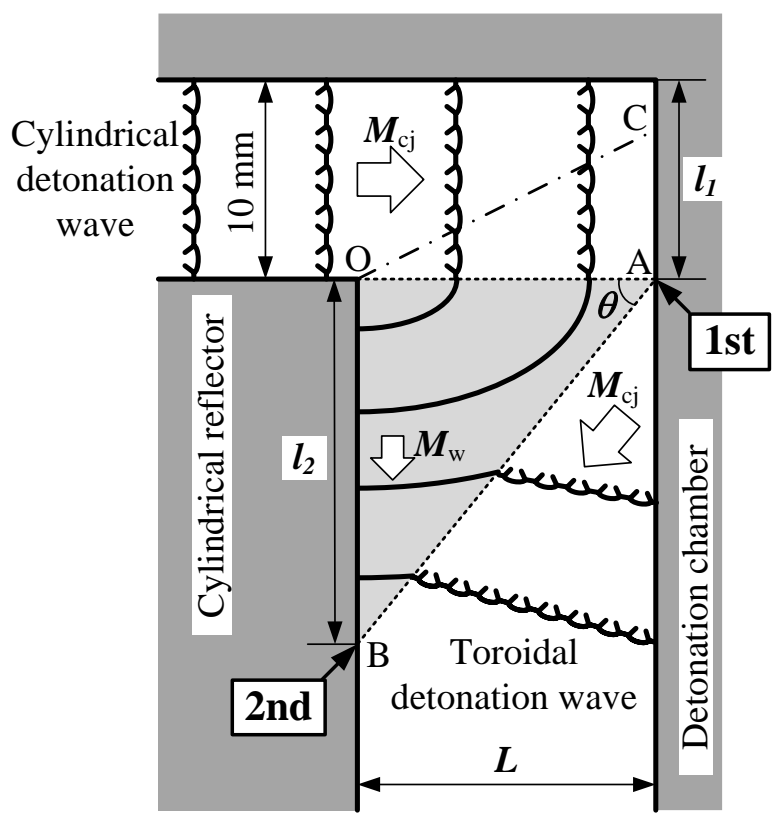

Fig. 7 Diffraction of the expanding cylindrical detonation wave around the edge of the reflector 


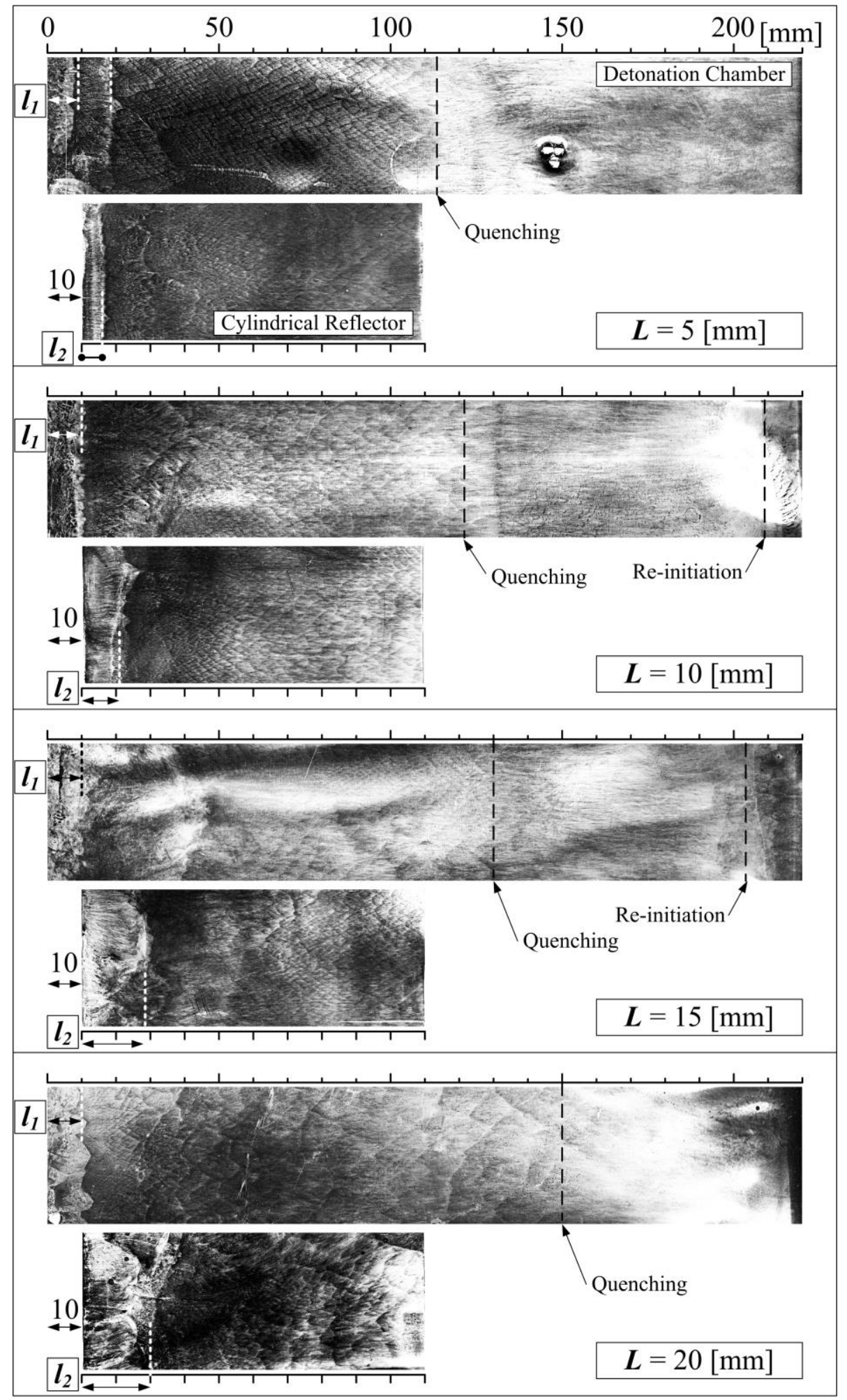

Fig. 8 Soot tracks on the sidewall of the detonation chamber and cylindrical reflector under the critical condition of transition to the planar detonation wave 


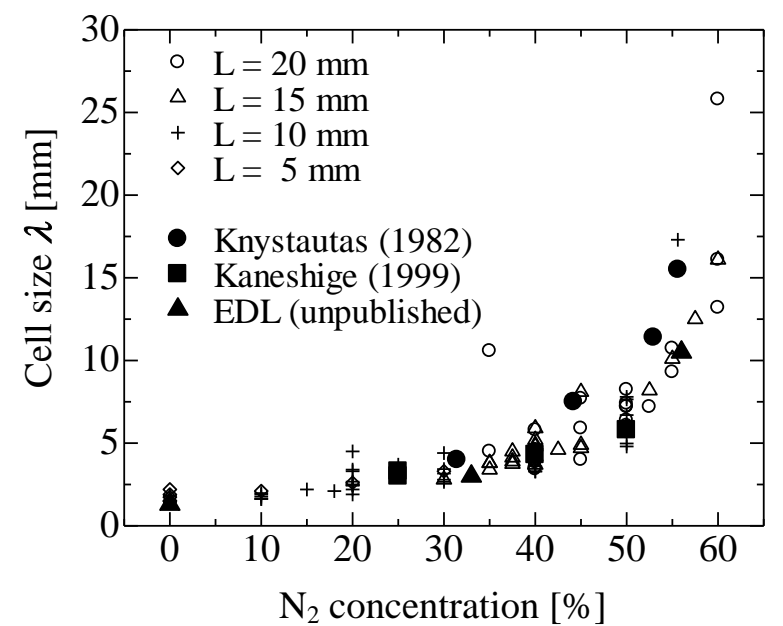

Fig. 9 Plot of the cell sizes obtained in the present study with previously reported results

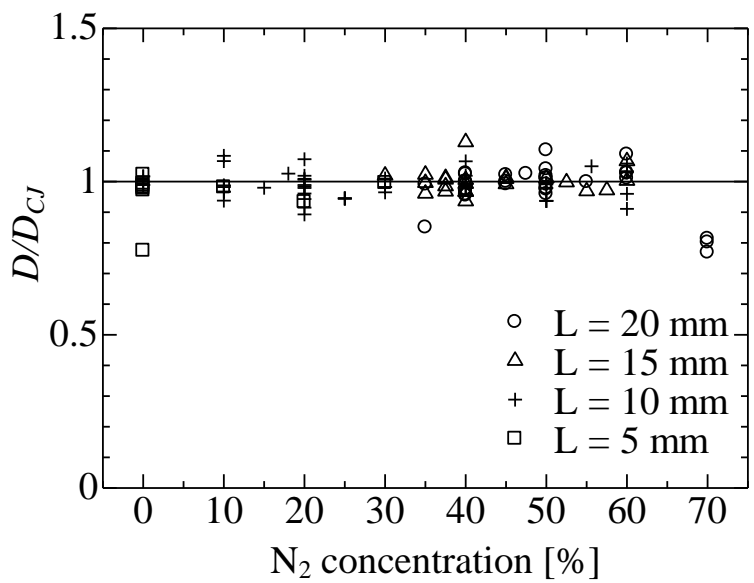

Fig. 10 Ratio of the detonation velocity $\boldsymbol{D}$ and theoretical value $\boldsymbol{D}_{c j}$ plotted against the nitrogen concentration 


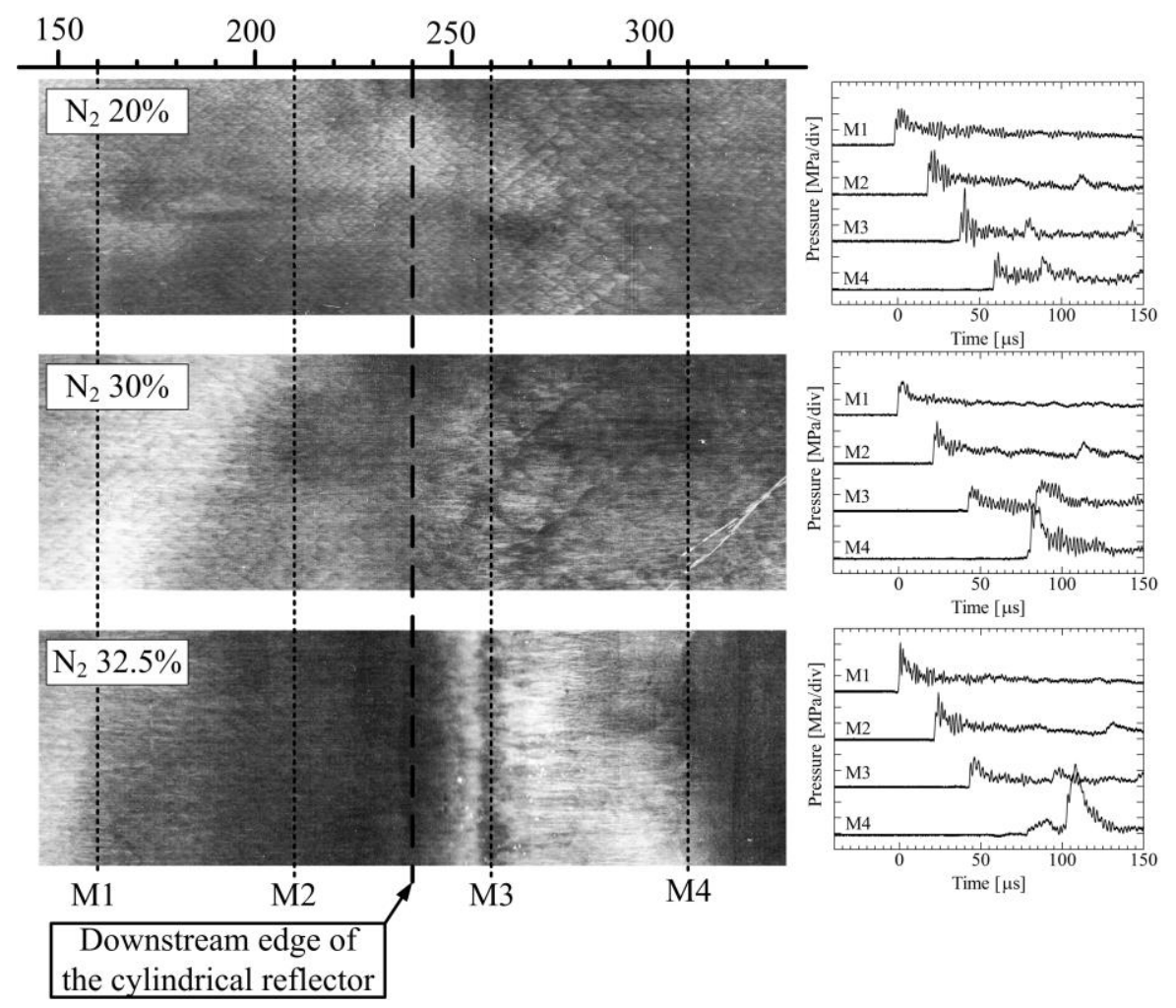

Fig. 11 Diffraction of the expanding cylindrical detonation wave around the edge of the reflector 


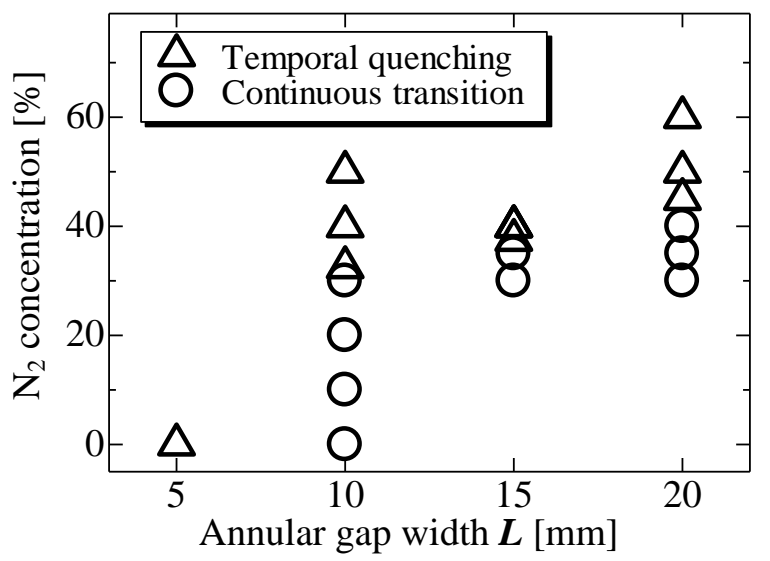

Fig. 12 Kinds of transitions for various $\mathrm{N}_{2}$ concentrations

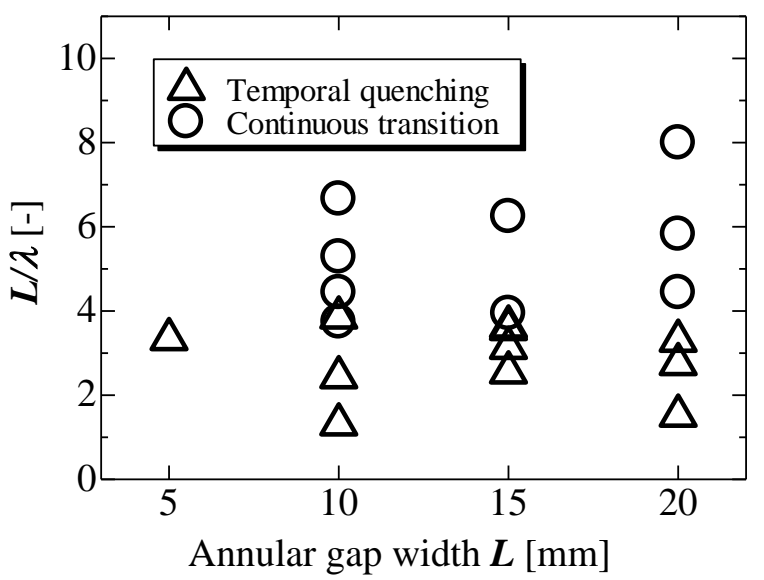

Fig. 13 Kinds of transitions vs. the ratio of the annular gap width and cell size 